\title{
Sclerotherapy complications of peripheral venous malformations
}

\section{Aronniemi, Johanna}

2016

Aronniemi , J , Castren , E , Lappalainen , K, Vuola , P , Salminen , P , Pitkäranta , A \&

Pekkola , J 2016 , ' Sclerotherapy complications of peripheral venous malformations ' , Phlebology , vol. 31 , no. 10 , pp. 712-722 . https://doi.org/10.1177/0268355515613740

http://hdl.handle.net/10138/229279

https://doi.org/10.1177/0268355515613740

publishedVersion

Downloaded from Helda, University of Helsinki institutional repository.

This is an electronic reprint of the original article.

This reprint may differ from the original in pagination and typographic detail.

Please cite the original version. 


\title{
Sclerotherapy complications of peripheral venous malformations
}

\author{
Johanna Aronniemi', Eeva Castrén², Kimmo Lappalainen', \\ Pia Vuola ${ }^{3}$, Päivi Salminen ${ }^{4}$, Anne Pitkäranta ${ }^{2}$ and \\ Johanna Pekkola'
}

\begin{abstract}
Background: Sclerotherapy is often the primary treatment for peripheral venous malformations. It is mostly sufficient alone, but can be combined with other endovascular techniques. Despite its mini-invasiveness, it is not without potentially severe complications. Here, we systematically report sclerotherapy complications in trunk and extremity venous malformations.

Methods: We retrospectively assessed the complications of 127 consecutive patients who had received sclerotherapy for peripheral venous malformation in our tertiary care unit (January 2007-August 2013). We applied the Clavien-Dindo classification to grade the severity of complications. We mostly used detergent sclerosants (85.7\%), and less often ethanol $(5.7 \%)$ or bleomycin (4.2\%). In $4.2 \%$ of the procedures, we combined glue, coils, endovascular laser or particles to sclerotherapy.

Results: The overall complication rate per procedure was 12.5\%. Most complications (83.3\%) were local and managed conservatively. We encountered four severe complications, all related to blood coagulopathy. Subcutaneous lesion location and use of ethanol significantly increased the risk of local complications.

Conclusion: Sclerotherapy alone or combined with other endovascular techniques is a safe method for local venous malformations with moderate risk for conservatively manageable complications. Blood coagulopathy constitutes a risk for, otherwise rare, severe complications.
\end{abstract}

\section{Keywords}

Venous malformation, sclerotherapy, complications, consumption coagulopathy, interventional radiology

\section{Introduction}

Venous malformations (VMs) are the most common slow-flow vascular malformations. They are present at birth and grow along with the child. Although at infancy they may be imperceptible, they have a tendency to expand as a result of any growth stimulus: hormonal, traumatic, or surgical. ${ }^{1}$ These morphologically heterogeneous lesions can occur in any part of the body and invade any tissue plane, which makes the symptoms diverse, varying from mild swelling to recurrent pain episodes, functional impairment, and life-threatening blood coagulopathy. Coagulation of stagnant blood in extensive lesions stimulates thrombin and initiates the conversion of fibrin to fibrinogen, which leads to localized intravascular coagulopathy (LIC). This entails a risk for both thrombosis and bleeding and can rarely progress to disseminated intravascular coagulopathy (DIC). ${ }^{2}$ In contrast to other vascular malformations, among trunk and extremity VMs, coagulation disorders can amount to $58 \% .^{3}$

In the past, the main treatment for VMs was surgery. In recent years, however, the generally preferred treatment has, in many centres, become percutaneous sclerotherapy. ${ }^{4-12}$ Extensive and diffuse lesions may not be surgically removable without severe

\footnotetext{
'Department of Radiology, University of Helsinki, HUS Medical Imaging Center, Helsinki University Central Hospital, Helsinki, Finland

${ }^{2}$ Department of Otorhinolaryngology-Head and Neck Surgery, University of Helsinki, Helsinki University Central Hospital, Helsinki, Finland

${ }^{3}$ Department of Plastic Surgery, University of Helsinki, Helsinki University Central Hospital, Helsinki, Finland

${ }^{4}$ Department of Pediatric Surgery, University of Helsinki, Children's Hospital, Helsinki University Central Hospital, Helsinki, Finland
}

\section{Corresponding author:}

Johanna Aronniemi, Department of radiology, $\mathrm{HUCH}$, Children's Hospital, Stenbäckinkatu II, 00290 Helsinki, Finland.

Email: johanna.aronniemi@hus.fi 
Table I. Studies concerning sclerotherapy complications in trunk and extremity venous malformations.

\begin{tabular}{|c|c|c|c|c|c|c|}
\hline References & Patients (N) & Age (y) & $\begin{array}{l}\text { Follow-up } \\
\text { (months) }\end{array}$ & Malformation & Sclerosant & $\begin{array}{l}\text { Complication } \\
\text { rate per } \\
\text { patient }(\%)\end{array}$ \\
\hline Lee et al. $^{23}$ & 87 & - & 24 mean & VM & ethanol & 28 \\
\hline Tan et al. $^{24}$ & 72 & 31.7 mean & 41 mean & VM & STS & 10 \\
\hline Orlando et al. ${ }^{25}$ & 39 & 24 median & 18 median & VM deep & ethanol & 12 \\
\hline Orlando et al. ${ }^{26}$ & 81 & 21 median & 18 median & VM superficial & ethanol & 21 \\
\hline Gulsen et al. $^{27}$ & 19 & 12.6 mean & 2 median & VM & polidocanol & 65 \\
\hline Schumacher et al. ${ }^{28}$ & 75 & 26 mean & - & VM & ethanol gel & 12 \\
\hline Hoque et al. $^{29}$ & 83 & I5.I mean & 12 median & VM & ethanolamine oleate & 5 \\
\hline Barbera et al. ${ }^{5}$ & 25 & 22 mean & & VM & foam, glue, coiling & 25 \\
\hline Zhang et al. ${ }^{15}$ & 138 & Children & 6 median & VM & ethanol, bleomycine & 29 \\
\hline Bowman et al. ${ }^{30}$ & 60 & 31.7 mean & 24 mean & VMAVM & $\begin{array}{l}\text { ethanol, sotradecol, particles, } \\
\text { n-butyl cyanoacrylate, } \\
\text { ethanolamine, onyx }\end{array}$ & 27 \\
\hline Present study & 127 & 21.5 median & 35 median & VM & $\begin{array}{l}\text { STS, polidocanol, ethanol, } \\
\text { bleomycine, doxicycline }\end{array}$ & 24 \\
\hline
\end{tabular}

VM, venous malformation; AVM, arteriovenous malformation; STS, sodium tetradecyl sulfate

disfigurement or functional impairment. Moreover, because sclerotherapy is less invasive and has better cost-effectiveness, it has become the method of choice, even for small lesions. Surgery still plays an adjunctive role, and supplementary sclerotherapy may enhance its effectiveness. ${ }^{13}$ Although not always curative, for most patients, sclerotherapy provides symptom relief. ${ }^{14}$

At sclerotherapy, the sclerosant is percutaneously injected into the VM's venous spaces with ultrasound or fluoroscopy guidance. It causes endothelial damage, leading to vessel-occlusion and lesion shrinkage. This occlusion occurs gradually, often necessitating serial treatments. Sclerotherapy often suffices alone, but can in selected cases be combined with other endovascular techniques in order to close very large venous spaces or to control the flow and drainage routes of the VM.

\section{Sclerosants}

Ethanol is the most potent agent causing instant endothelial damage and effective vascular closure. In addition, ethanol can diffuse into nearby structures and cause variable degrees of tissue necrosis. Because of its high toxicity, it can cause the most serious sideeffects and should be used with caution and only by experienced practitioners. ${ }^{8,15}$

Detergent sclerosants, sodium tetradecyl sulphate (STS) and polidocanol, are less potent than ethanol, but also lower risk for local and systemic complications. Detergents also cause endothelial damage, inflammation, and fibrosis, but the sclerosing process occurs more slowly, with possibly a higher tendency toward recanalization. ${ }^{16}$ They offer, however, an effective and less toxic alternative to ethanol with different concentrations and forms (solution and foam) available. Microfoam, made by mixing the detergents with air or $\mathrm{CO}_{2}$, is more effective than liquid sclerosants. ${ }^{12,16}$ Additionally, STS and polidocanol cause less pain than ethanol, which reduces the need for general anaesthesia.

Bleomycin was originally approved for oncological chemotherapies and has since served to control excessive pleural effusions, as well. Like detergents, bleomycin has less scerosing potency than ethanol, but has the advantage of causing relatively little swelling. The chemotherapy-related tendency of bleomycin to cause pulmonary side-effects has long limited its usage. When treating VMs, however, bleomycin is administered intralesionally at much lower doses than for chemotherapy (15 units per procedure, not exceeding a cumulative dose of 300 units). At such a dosage, no reported cases of pulmonary fibrosis have occurred. ${ }^{16}$

\section{Complications}

Few articles report sclerotherapy complications in trunk and extremity VMs (Table 1). Most reported complications are minor or local, but also systemic complications exist. ${ }^{17}$ Common sclerotherapy complications are transient hemoglobinuria and oliguria. ${ }^{18}$ Furthermore, single case-reports cover sclerotherapyrelated pulmonary embolism, ${ }^{19}$ reversible cardiac arrest after polidocanol sclerotherapy, ${ }^{20}$ fatal cardiovascular collapse during ethanol sclerotherapy, ${ }^{21}$ and extensive soft-tissue necrosis and infection after STS and ethanol sclerotherapy. ${ }^{22}$ 
Table 2. Clavien-Dindo classification for surgical complications. ${ }^{31,32}$

\begin{tabular}{|c|c|}
\hline Management of complication & Grade \\
\hline $\begin{array}{l}\text { Any deviation from normal postoperative course without need for pharmacological treatment or } \\
\text { surgical, endoscopic, or radiological interventions. Acceptable therapeutic regiments are: drugs } \\
\text { as antiemetics, antipyretics, analgetics, diuretics, electrolytes, and physiotherapy. Also includes } \\
\text { wound infections opened at bedside }\end{array}$ & I \\
\hline Requiring pharmacological treatment with drugs other than acceptable for grade I & II \\
\hline Requiring surgical, endoscopic, or radiological intervention & III \\
\hline Intervention not under general anesthesia & Illa \\
\hline Intervention under general anesthesia & Illb \\
\hline Life-threatening complication requiring intermediate care/intensive care-unit management & IV \\
\hline Single-organ dysfunction (incl. dialysis) & $\mathrm{IVa}$ \\
\hline Multiorgan dysfunction & $\mathrm{IVb}$ \\
\hline Death of patient & $\mathrm{V}$ \\
\hline
\end{tabular}

Our multidisciplinary team (MDT) uses the Clavien-Dindo complication classification, ${ }^{31-33}$ originally developed to assess surgical outcomes. Use of the same classification for sclerotherapy makes comparison between these two treatment modalities possible, also in terms of complications. The Clavien-Dindo classification grades complications on the basis of further treatment needed to manage the complication. It divides complications into conservatively manageable grades I and II, into surgical, radiological, or endoscopic intervention-requiring grade III, into life-threatening grade IV, and fatal grade $\mathrm{V}$ complications (Table 2).

To improve the safety of any treatment, systematic assessment and reporting of complications are essential. This reporting must be accurate and objective, avoiding any subjective descriptions. Our aim is to report on complications of endovascular treatment for trunk and extremity VMs and to assess variables affecting the complication rate.

\section{Materials and methods}

\section{Patients}

This retrospective study was approved by the Ethics Committee of Helsinki University Central Hospital. We reviewed all 127 patients with trunk or extremity VM undergoing sclerotherapy over a period of 6 years and 8 months (from 1 January, 2007 to 31 August 2013) at Helsinki University Central Hospital, a tertiary referral centre providing care for 1.5 million people. We defined lesion location and tissue planes involved from the magnetic resonance imaging (MRI) and ultrasonography (US) studies and went through patient records to assess complication details, follow-up time, and possible surgical treatment.
The multidisciplinary team for vascular anomalies, established in 2002, evaluated each patient and made recommendations for treatment. The diagnosis was based on the clinical picture and imaging studies according to the International Society for the Study of Vascular Anomalies (ISSVA) 1996 classification. ${ }^{34}$ Indications for sclerotherapy were pain, functional impairment, swelling, skin induration, and blood coagulopathy, cosmetic indications being few. Asymptomatic patients and patients with only mild symptoms were followed up or treated conservatively by the means of compression garments.

\section{Sclerotherapy procedure}

The sclerosants used in the majority of cases were STS 3\% (Fibrovein ${ }^{\circledR}$, STD Pharmaceutical, Hereford, UK) and polidocanol $0.5-3 \% \quad$ (Aethoxysklerol $^{\circledR}$, BASE Pharma, Canberra, Australia) (Table 4). In addition, we used ethanol $95 \%$ and bleomycin $1 \mathrm{mg} / \mathrm{ml}$ (Bleomycin ${ }^{\circledR}$, Baxter, Halle/Westfalen, Germany) as sole agents or combined with other sclerosants, and once doxycycline $10 \mathrm{mg} / \mathrm{ml}$ (Doxy $100^{\circledR}$, Fresenius Kabi USA, LLC) for a suspected lymphatic malformation that turned out during the procedure to be venous. The use of ethanol radically diminished during the study period. We selected the sclerosant according to lesion location, depth, and size. We used lower concentrations $(0.5-1 \%)$ of polidocanol for superficial lesions and STS 3\% and polidocanol 3\% for deeper lesions. STS and polidocanol were used as foam. We made the foam by mixing the sclerosant with air (or $\mathrm{CO}_{2}$ ) between two syringes $(5$ or $10 \mathrm{ml})$ through a threeway stopcock on average 20 times. The liquid-air fraction was typically $1 / 3-4$, but was not systematically determined. For wide venous spaces, the foam was prepared with proportionally more air. The aim was to 


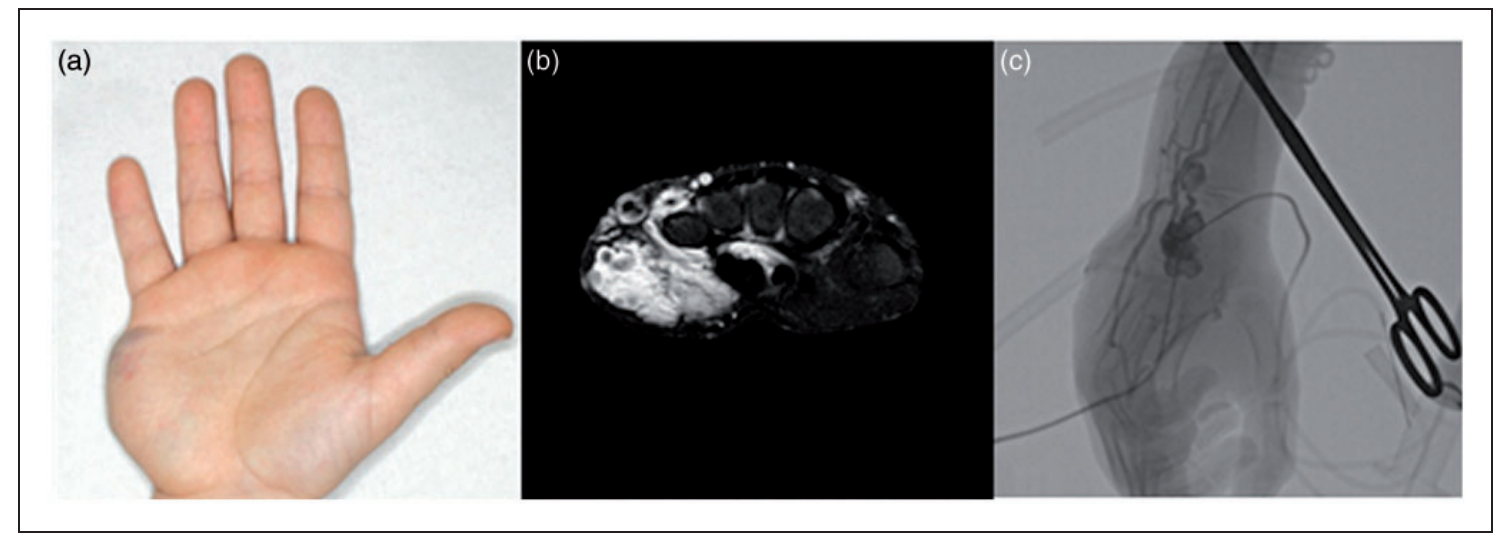

Figure I. Venous malformation in the hand of a 7-year-old boy. (a) The hand before sclerotherapy has a prominent mass in the hypothenar. (b) T2-weighted fat-saturated axial magnetic resonance image clearly shows pathological venous structures with phlebolites and defines lesion extent and tissue planes involved. (c) Fluoroscopy image prior to percutaneous sclerotherapy by doubleneedle technique.

achieve as large contact area as possible between the surfactant molecules and endothelium and to completely fill the venous spaces. The quality of the foam was visually checked before injection, and the foam was used immediately after the generation.

In most procedures $(95.8 \%)$, we only used percutaneous sclerotherapy. Other endovascular techniques, such as n-butyl-2-cyanoacrylate (NBCA) glue (Histoacryl ${ }^{\circledR}$, B.Braun, Melsungen, Germany), coils (Interlock ${ }^{\circledR}$, Boston Scientific, Boston, or Azur ${ }^{\circledR}$, Terumo, Somerset, NJ, USA), particles (Bead Block ${ }^{\circledR}$, Terumo, Somerset, NJ or Embozene ${ }^{\circledR}$, Celo Nova, San Antonio, TX, USA), or intravascular laser, were combined to sclerotherapy in $4.2 \%$ of the procedures. The indication for using NBCA glue together with foam sclerosants was closing very large venous spaces, for which sclerotherapy alone was considered insufficient. In selected cases, we used coils for closing the VM's draining veins prior to sclerosant administration - for example, if the fluoroscopy showed sizeable communication of the VM with the extremity deep venous system. This was to prevent the sclerosant from disseminating to the normal venous system. Coils were not deployed into the VM itself. We utilized arterial PVA embolization together with intralesional foam sclerotherapy for one patient to close feeding arteries of the VM and intravascular laser combined to glue and sclerosant for another patient with extremely wide VM. For the two laser procedures, we utilized radial laser (Biolitec, Jena, Germany) with $10 \mathrm{~W}$ power and $1470 \mathrm{~nm}$ continuous wave. The fiber was inserted to the malformation through 6 frence introducers at several points. The energies used were from 4600 to $5200 \mathrm{~J}$ and the withdrawal rate was very slow.

We controlled the blood d-dimer and fibrinogen prior to the procedure and treated any coagulopathy in cooperation with a haematologist. Each patient with extremity lesions was provided with an individually customized compression garment.

Our sclerotherapy approach closely parallels that described by Alomari et al. ${ }^{35}$ Two interventional radiologists performed the procedures. We did the procedures in sterile conditions under local or general anaesthesia or under intravenous conscious sedation, depending on patient age, cooperation, and anticipated pain. For extremity lesions, we first applied a tourniquet proximal to the lesion to make the venous structures more visible and to control venous outflow. We cannulated the lesion, under US guidance, with small needles. After confirming free return of venous blood from the needle, we injected iodinated contrast under fluoroscopic control to ensure proper intravenous access and to define the extent of the lesion and the potential draining veins. This also gives an estimate of the volume needed to fill the lesion. In cases with rapid venous outflow or drainage through potentially dangerous routes, we compressed the draining veins by hand or tourniquet or if necessary occluded them intravascularly with coils before sclerosant injection. We typically used a double-needle technique, in which we let blood and the agent exit through a second needle placed elsewhere in the lesion. ${ }^{11}$ This prevents lesion overfilling and ensures that the whole lesion is drained (Figure 1).

To reduce swelling and to facilitate vessel occlusion in extremity lesions, we placed a compression garment (23-32 $\mathrm{mmHg}$ ) over the injection site after the procedure. This was typically worn for three days continuously and two weeks during physical activity. We monitored urine haemoglobin and volume in cases with extensive lesions. ${ }^{36}$ We did not administer steroids routinely. We used them only during treatment of intramuscular VMs within confined muscular compartments, in order to diminish swelling and lessen the 
risk for muscle compartment syndrome (iv. dexamethasone perioperatively and po. prednisolone for 2-4 days with decreasing doses). We typically discharged the patient on the same or the following day and decided each follow-up schedule individually. If no further interventions or follow-up visits were scheduled, we encouraged the patient if symptoms returned to contact us.

\section{Definition and classification of complications}

We designated as complications all disabilities that developed as a consequence of our treatment. Almost all patients, however, experienced some postprocedural pain or swelling, which we considered an expected sideeffect, not a complication, if lasting less than two weeks. Nor was failure to cure considered a complication. We graded complications according to the Clavien-Dindo classification (Table 2).

\section{Statistical analysis}

An independent statistician analysed the data with NCSS 8 statistical software. The Chi-Square test or Fisher's Exact test was performed for variables possibly predicting complications (age, lesion location, tissue plane, sclerosants, and preceding surgery). The Mann-Whitney $U$ test served for analysis of the dependence between number of procedures and complications. For comparison of risks presented by different sclerosants, we conducted a chi-square test between two groups: a combined detergent group (STS and polidocanol) and an ethanol group (ethanol as the sole agent or in combination with any other sclerosants). We considered a probability level of $p<0.05$ as statistically significant.

\section{Results}

Table 3 shows descriptive data of the patients $(n=127$, age median 21.5) and procedures $(n=280$, median 2.0). Lesion size ranged from small subcutaneous lesions to ones so extensive and diffuse that they affected the whole retroperitoneum or extremity.

Thirty-one $(24.4 \%)$ patients had a complication with the overall complication rate per procedure being $12.5 \%$. Three patients developed a complication after each of two different procedures, and one patient developed two independent complications (allergic reaction and bleeding) after one procedure.

The tissue plane involved influenced the complication rate: risk was significantly higher in subcutaneously confined lesions than in deeper lesions with an intramuscular component $(p=0.049)$. The age of the patient ( $\leq 17$ vs $>17$ years), number of procedures,
Table 3. Characteristics of patients and treatments.

\begin{tabular}{|c|c|}
\hline & $N(\%)$ or median (range) \\
\hline Patients & 127 \\
\hline Female & $73(57.5)$ \\
\hline Male & $54(42.5)$ \\
\hline Age median (range) & $21.5(3.4-76.0)$ \\
\hline$\leq 17$ years & $47(37.0)$ \\
\hline$>17$ years & $80(63.0)$ \\
\hline \multicolumn{2}{|l|}{ Diagnosis } \\
\hline MRI & 118 (92.9) \\
\hline histology & II (8.7) \\
\hline only US & $8(6.3)$ \\
\hline \multicolumn{2}{|l|}{ Lesion location } \\
\hline Lower extremity & $62(48.8)$ \\
\hline Upper extremity & $50(39.4)$ \\
\hline Trunk & $15(1 \mathrm{I} .8)$ \\
\hline \multicolumn{2}{|l|}{ Tissue plane } \\
\hline Subcutaneous & $59(46.5)$ \\
\hline Intramuscular & $64(50.4)$ \\
\hline Intraosseal & $5(3.9)$ \\
\hline Intra-articular & $4(3.2)$ \\
\hline Intra-abdominal, retroperitoneal & $2(1.6)$ \\
\hline \multicolumn{2}{|l|}{ Surgery } \\
\hline Previous surgery & $29(22.8)$ \\
\hline Surgery after sclerotherapy & $10(7.9)$ \\
\hline Sclerotherapy procedures & 280 \\
\hline Procedures per patient & $2.0(1-10)$ \\
\hline Follow-up, months & $35.0(0-82)$ \\
\hline Last visit, months & $19.0(0-80)$ \\
\hline
\end{tabular}

MRI, magnetic resonance imaging; US, ultrasound.

or location of the malformation treated did not significantly affect complication risk. Preceding surgical excision (29 patients) did not elevate the probability of sclerotherapy complications, nor did any sclerotherapy complication predict further need for surgical treatment.

Table 4 shows complication numbers from each sclerosant. Detergent sclerosants were the choice in the majority of procedures $(85.7 \%)$, with equal complication rates from STS $(11.1 \%)$ and polidocanol $(11.8 \%)$. Bleomycine was only used in four procedures and caused no complications. Ethanol served as the sole agent or in combination with other sclerosants in 16 procedures with a complication rate of $31.2 \%$, which was significantly higher $(p=0.019)$ than in the combined-detergent group (STS and polidocanol).

We combined one or several endovascular techniques to percutaneous sclerotherapy for eight patients in 12 procedures; glue in 10 , coils in 4 , intravascular laser in 3, and particles in one procedure. 
Table 4. Complication numbers for the sclerosants.

\begin{tabular}{lcc}
\hline Sklerosant & Procedures $(N)$ & Complications $(N)$ \\
\hline STS 3\% & 189 & 21 \\
Polidocanol 0.5-3\% & 51 & 6 \\
Ethanol 95\% as sole agent & 3 & 2 \\
Ethanol alone or in & 16 & 5 \\
$\quad$ combination & & 0 \\
Bleomycine as sole agent & 4 & 1 \\
Bleomycine alone or in & 12 & \\
$\quad$ combination & & 1 \\
Doxycycline & 1 & \\
\hline
\end{tabular}

STS, Sodium tetradecyl sulphate.

A complication occurred in three of these procedures where we combined sclerotherapy with glue or with glue and intravascular laser. All the three complications were related to blood coagulopathy with an additional infection in two of them. No complications followed the use of coils or particles.

According to the Clavien-Dindo classification, most complications (30 cases, $83.3 \%$ ) were conservatively managed grade I-II (Table 5), of which the most significant were local skin damage $(n=12)$ and prolonged pain or swelling at the injection site $(n=9)$. We encountered two deeper skin necrosis, which necessitated skin grafts (grade IIIa), and additionally, three grade IV and one grade $\mathrm{V}$ complication to be discussed in detail. Figure 2 shows a grade IIIa complication.

The four grade IV-V complications involved the three patients with the most complicated and diffuse VMs (Figure 3). Moreover, in all these patients, the malformation caused severe consumption coagulopathy.

One paediatric patient (Figure 3, top panel) with an extensive and diffuse retroperitoneal VM, after dorsal needle puncture of the retroperitoneal lesion, suffered from severe (6 1) intra-abdominal bleeding from the left phrenic artery. He underwent intravascular embolization and recovered after treatment in an intensive care unit. The main indication for treatment was consumption coagulopathy and DIC which is, after several sclerotherapy procedures, now under control.

The man in Figure 3 (middle panel) had an extremely extensive VM of the right leg and lower body, causing hygienic and life quality problems. He had severe consumption coagulopathy, a fibrinogen level close to zero, and episodes of life-threatening bleedings and pulmonary embolism. The malformation was considered inoperable because of risk for uncontrollable bleeding. The sclerotherapy-related risk for skin damage and infection was high and was thoroughly discussed. Coagulopathy and facilitation for further surgery were the main indications for sclerotherapy.
After two separate sclerotherapy sessions, he had severe infection complications. The first time, after sclerotherapy with polidocanol and ethanol combined with NBCA and laser therapy, he bled at the injection site and had skin damage followed by infection of the haematoma and sepsis. He was treated with intravenous antibiotics and prolonged hospitalization in an intermediate care unit. The second time, after perirectal foam sclerotherapy combined with NBCA and laser therapy, he experienced worsening of the DIC, bleeding, sepsis, and abscess formation. He recovered from both severe infections and has thereafter undergone several sclerotherapy procedures without complications. His coagulopathy is now under control, the malformation mass has shrunk, and surgical treatment to reduce the mass is scheduled.

The woman in Figure 3 (bottom panel) had a wide and diffuse VM covering her entire upper extremity and axilla. This malformation caused consumption coagulopathy and DIC with chronic need for low-molecular weight heparin. The main indication for treatment was that the affected limb was totally unusable, and the goal was to facilitate further surgery. The procedure was carefully planned, and the coagulation disorder was monitored and treated by a haematologist prior to and during the procedure. The patient underwent sclerotherapy with percutaneous STS 3\% and NBCA glue. Despite all precautions and treatment of the coagulopathy, the following day she suffered a multifocal intracerebral haemorrhage, which was eventually fatal. She had had no previous neurological symptoms or imaging examinations of the brain, and neuropathological autopsy revealed no predisposing condition for intracerebral haemorrhage.

\section{Discussion}

Our experience indicates that the major risk for severe complications of the VMs endovascular treatment is blood coagulopathy. This has not been systematically reported earlier, although the association of VMs with blood coagulopathy is well documented., ${ }^{2,3,37}$ All the most serious (grade IV and V) complications were, at least in part, due to blood coagulopathy. Less severe (grade I-III) complications were associated with superficial lesions and use of ethanol.

Most of the coagulopathy related complications occurred in procedures with other endovascular techniques (glue or/and laser) combined to percutaneous sclerotherapy. This is obviously due to the fluctuation of coagulation factors during the treatment of a wide VM and may not be associated with any specific treatment modality. The infection complications, otherwise very rare in sclerotherapy, were related to the use of NBCA glue and laser, for which the probable cause 
Table 5. Descriptions and numbers of complications and details of necessitated managements. Complications classified according to Clavien-Dindo (Table 2).

\begin{tabular}{|c|c|c|c|}
\hline Complication & Management & $N$ & Grade \\
\hline Skin damage & Not necessitated & 8 & I \\
\hline Pain or swelling or both for $>2$ weeks & Analgesics and/or compression garment & 6 & I \\
\hline Thrombophlebitis outside the malformation & Not necessitated & 3 & 1 \\
\hline Unusual pain and transient sensory loss & Analgesics & I & I \\
\hline Unusual swelling and loss of range of joint motion & Physiotherapy & I & I \\
\hline Pain, swelling, skin damage, and loss of range of motion & Analgesics, physiotherapy & 1 & I \\
\hline Digital loss of range of joint motion & Physiotherapy & I & I \\
\hline Skin damage, infected & Antibiotics, per oral & 4 & II \\
\hline Allergic reaction & Corticosteroids, antihistamine & 3 & II \\
\hline Intra-abdominal bleeding, mild & Blood transfusion (one unit) & 1 & II \\
\hline Deep vein htrombosis at ankle level & LMWH medication & 1 & II \\
\hline Skin damage & Skin graft under local anesthesia & 2 & Illa \\
\hline $\begin{array}{l}\text { Bleeding at injection site, sepsis, abscess, } \\
\text { worsening of DIC }\end{array}$ & $\begin{array}{l}\text { Transfusions of blood and coaculation factors, } \\
\text { i.v antibiotics, abscess drainage, ICU treatment } \\
\text { and prolonged hospitalization }\end{array}$ & I & IV \\
\hline Bleeding at injection site, skin damage and sepsis & $\begin{array}{l}\text { Intermediate care-unit treatment, i.v. antibiotics, } \\
\text { long hospitalization, }\end{array}$ & 1 & IV \\
\hline Intra-abdominal bleeding (6 liters) & $\begin{array}{l}\text { Blood transfusion, intra-arterial embolisation, } \\
\text { ICU treatment }\end{array}$ & I & IV \\
\hline Intracerebral hemorhage and exitus & & I & $\mathrm{V}$ \\
\hline
\end{tabular}

LMWH, low-molecular-weight heparin; ICU, intensive care unit; DIC, disseminated intravascular coagulopathy.

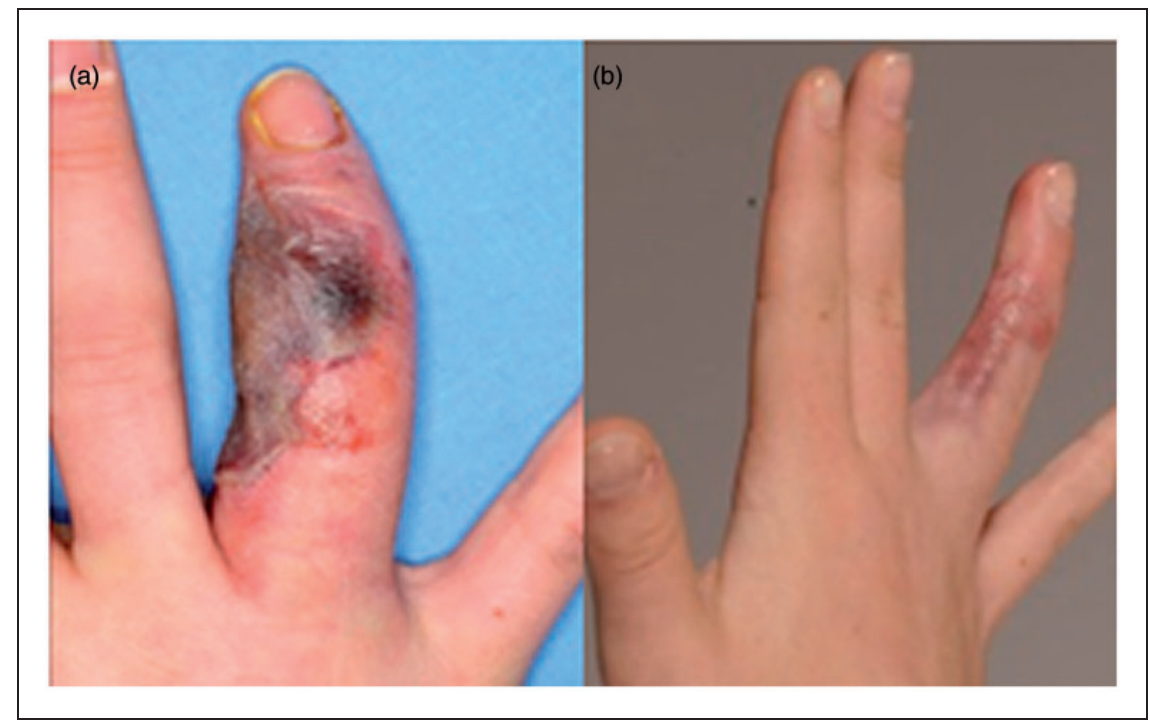

Figure 2. A previously operated venous malformation with recurrence in the right fourth finger. (a) Skin necrosis after sclerotherapy with sodium tetradecyl sulphate $3 \%$ necessitated skin grafting and was graded as Illa due to the need for surgical treatment. (b) The same finger three months after skin grafting.

was the glue being a foreign material. We had no complications associated to the coiling of draining veins.

A consequence of the infection complications has been further development of our treatment guidelines.
We now use antibiotic prophylaxis for patients with pre-existing skin damage, with an apparent risk for skin damage or when using glue to close large venous spaces. In these cases, the foreign material with clotted 

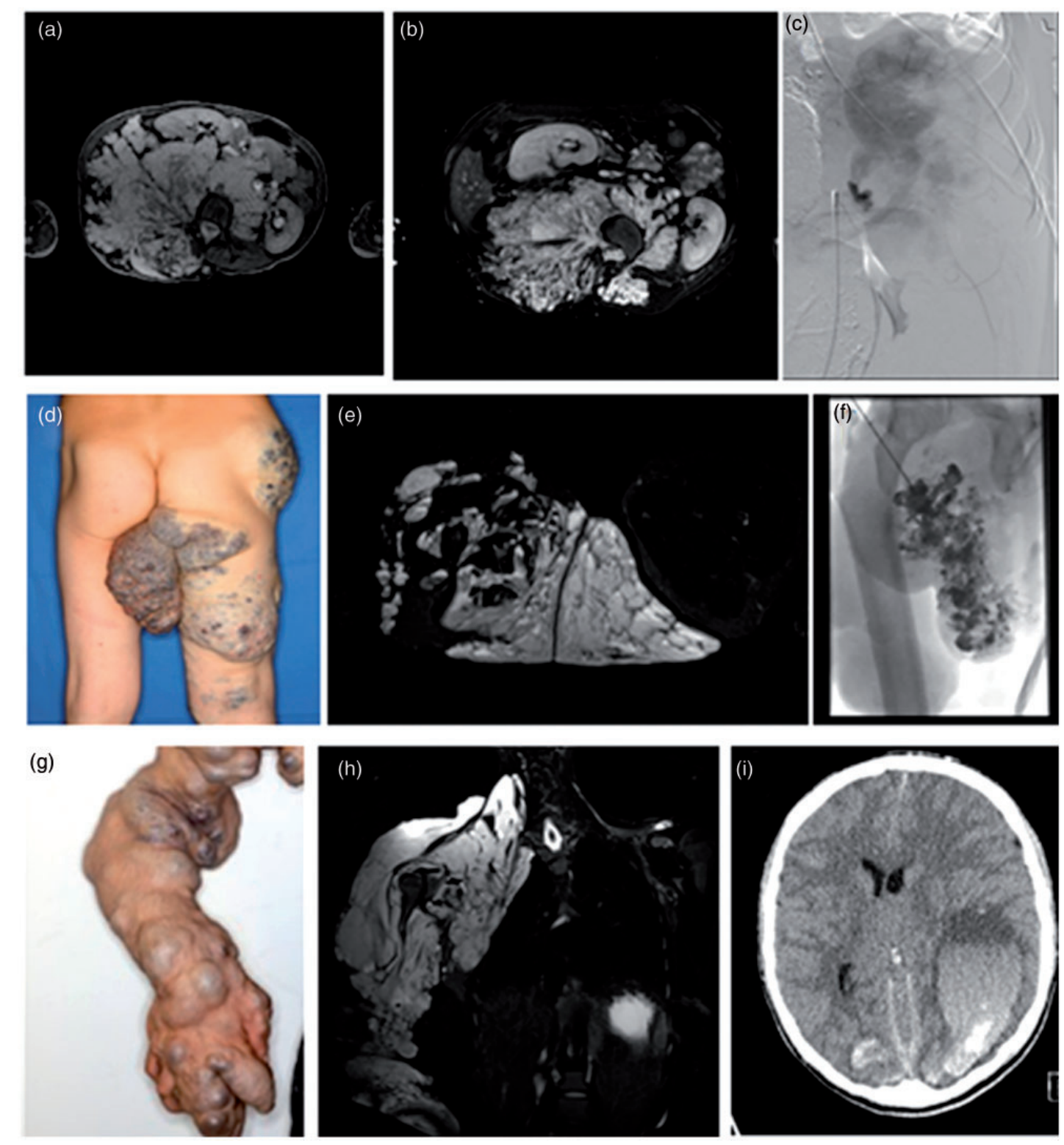

Figure 3. The three patients facing the most severe grade IV to $V$ complications. Top panel: $A$ 9-year-old boy with an extensive and diffuse venous malformation (VM) of the retroperitoneum, paraspinal muscles, and spinal canal. VM dislocates the right kidney anteriorly and causes mild hydronephrosis in the left kidney. T2-weighted fat-saturated axial magnetic resonance (MR) image before (a) and after (b) several procedures shows reduction of the mass. Angiography image of bleeding from the phrenic artery, complicating sclerotherapy of the lesion (c). Middle panel: A 45-year-old man with extensive infiltrating VM in the pelvis, perineum, and right thigh. A large malformation mass hangs from the perineum (d). Extent of the lesion in T2-weighted fat-saturated axial MR image (e). Fluoroscopy image of the sclerotherapy procedure reveals wide venous structures filled with contrast material ( $f$ ). Bottom panel: A 32-year-old woman with extensive VM involving the entire right arm and axilla (g). T2-weighted fat-saturated coronal MR image shows the lesion extending into the thorax cavity (h). CT image of a fatal multifocal intracerebral haemorrhage after the sclerotherapy procedure (i).

blood increases the risk for infection. Treatment of extensive perirectal or perineal malformations may require a temporary colostomy. Cooperation with a plastic surgeon is essential in treating soft-tissue complications.
Treatment of patients with extensive VMs should be pre-planned in a multidisciplinary setting and performed in centres with a haematology consultant available, possibility to monitor and treat the coagulation disorders before, during, and after the procedure and, 
when necessary, the available option of ICU treatment. This concerns, however, only a small minority of VMs.

The total complication rate in our tertiary care unit ( $12.5 \%$ of the procedures and $24.4 \%$ of the patients) corresponded to earlier figures (Table 1). Reported complication rates vary greatly, however, depending on sclerosants, patient selection, and definition of complication, which makes comparison difficult. Gulsen et $\mathrm{al}^{27}$, for example, report polidocanol sclerotherapy data with a complication rate of $65 \%$, considering pain and swelling as minor complications, while Tan et al. ${ }^{24}$ report, for STS sclerotherapy, a complication rate of only $9.7 \%$. Our complication rates for each of these two sclerosants were equal $(11 \%)$. The complication risk has been significantly higher with ethanol than with bleomycine. ${ }^{15} \mathrm{We}$ showed the risk from ethanol as being significantly higher also than that of detergents which are now commonly used sclerosants in many centers. Compared to deep lesion location, subcutaneous location constituted a higher complication risk with both ethanol and detergent sclerosants; this corresponded to others' findings with ethanol. ${ }^{25,26}$

The high variability of complication rates is apparently due to the lack of uniform definition and classification of complications. Many of the reports apply no classification at all, ${ }^{5,15,25,29,30}$ or they divide complications into local and systemic ${ }^{28}$ or major and minor, ${ }^{23,24,27}$ without clearly defining these descriptions. The Clavien-Dindo classification, applied in this study, is previously used in one report on head and neck VM sclerotherapy complications. ${ }^{33}$

The risk for skin damage is higher in superficial than in deeper lesions. Although it can be reduced by using low concentration polidocanol foam, these complications still occur. They mostly heal well, but cause transient discomfort and scarring. This emphasizes the importance of patient counselling: each patient must know the risk for skin damage beforehand, and risk should be carefully weighed against symptoms. ${ }^{35}$ When evaluating indications for treatment, one essential factor is correct diagnosis. For example, two of our patients with prolonged pain and swelling as complications, obviously had fibro-adipose vascular anomaly (FAVA) ${ }^{38}$; based on our current knowledge, they should have received no sclerotherapy.

An experimental study with rat femoral vein model has shown that in intraluminal administration, variable amounts of sclerotic agents leak in into surrounding tissues, which may have detrimental effects on adjacent nerves. ${ }^{39}$ This entails risk for neural damage, especially in restricted muscle compartments. We had, however, only a single neural complication, which was transient sensory loss in one finger. In a systematic review of 1214 patients, the risk for neural damage after VM sclerotherapy was $1.85 \%{ }^{40}$ Contrary to common belief, neural complications are thus fairly rare.

In patients undergoing several sclerotherapy procedures or previous surgical treatment, the complication rate showed no increase, nor did patient age or malformation location influence complication rate. Complications thus seem to be independent events influenced mainly by lesion morphology and depth. The relatively low patient number, however, amplifies uncertainty regarding these negative results.

Evaluation of treatment outcomes was beyond the scope of this study. It turned out to be unreliable based on patient records and thus deserves its own prospective study.

Study limitations were mainly related to its retrospective nature. Severity and duration of pain and swelling were difficult to assess on the basis of patient records. Typically, the patient had had similar symptoms prior to the treatment, and whether they were exacerbated by the sclerotherapy was not evident in every case. During the study period, we have used a wide variety of devices such as different needles, syringes, catheters, introducers, and vascular access kits. The selection of equipment has based on the specific anatomy of each lesion and the availability of the equipment over the years. We were not able to specify the source of all equipment used. Any methodological weaknesses could be lessened by collecting data on the patients and the procedures systematically and prospectively. For these purposes, we are now setting up a patient registry for vascular anomalies with automated data collection. Moreover, the confidence regarding risks with various sclerosants was limited by numbers of patients and procedures.

Finally, one should note that a sclerotherapy complication does not necessarily mean unsuccessful treatment, and despite any complication, patients often consider the overall outcome as satisfactory. Regarding the risk for complications, all VMs do not necessarily have to be treated, especially those causing only mild cosmetic harm. On the other hand, some malformations should only be treated in centres prepared to manage the potentially very severe complications. Benefits and risks must be weighed in each case in a multidisciplinary setting.

\section{Acknowledgement}

We thank Carol Norris, $\mathrm{PhD}$, for reviewing the language.

\section{Declaration of Conflicting Interests}

The author(s) declared the following potential conflicts of interest with respect to the research, authorship, and/or publication of this article: The authors of this manuscript declare no relationships with any companies, whose products or services may be related to the subject matter of the article. 


\section{Funding}

The author(s) disclosed receipt of the following financial support for the research, authorship, and/or publication of this article: This study has received funding by the state funding for university-level health research.

\section{Research ethics}

The study is approved by the local ethics committee (Helsinki University Central Hospital, Operative ethics committee, reference number 319/13/03/02/2011).

\section{References}

1. Lee B and Laredo J. Venous malformation: treatment needs a bird's-eye view. Phlebology 2013; 28: 62-63.

2. Dompmartin A, Acher A, Thibon P, et al. Association of localized intravascular coagulopathy with venous malformations. Arch Dermatol 2008; 144: 873-877.

3. Mazoyer E, Enjolras O, Bisdorff A, et al. Coagulation disorders in patients with venous malformation of the limbs and trunk: a case series of 118 patients. Arch Dermatol 2008; 144: 861-867.

4. Cabrera J, Cabrera J Jr and Garcia-Olmedo MA. Sclerosants in microfoam. A new approach in angiology. Int Angiol 2001; 20: 322-329.

5. Barbera L, Fiedler HW and Krauss M. The embolisation of venous malformations. Zentralbl Chir 2012; 137: 485-490.

6. Dompmartin A, Vikkula M and Boon LM. Venous malformation: update on aetiopathogenesis, diagnosis and management. Phlebology 2010; 25: 224-235.

7. Khandpur S and Sharma VK. Utility of intralesional sclerotherapy with $3 \%$ sodium tetradecyl sulphate in cutaneous vascular malformations. Dermatol Surg 2010; 36: $340-346$.

8. Legiehn GM and Heran MK. A step-by-step practical approach to imaging diagnosis and interventional radiologic therapy in vascular malformations. Semin Intervent Radiol 2010; 27: 209-231.

9. Odeyinde SO, Kangesu L and Badran M. Sclerotherapy for vascular malformations: complications and a review of techniques to avoid them. J Plast Reconstr Aesthet Surg 2013; 66: 215-223.

10. Hassanein AH, Mulliken JB, Fishman SJ, et al. Venous malformation: risk of progression during childhood and adolescence. Ann Plast Surg 2012; 68: 198-201.

11. Burrows PE. Endovascular treatment of slow-flow vascular malformations. Tech Vasc Interv Radiol 2013; 16: 12-21.

12. Rabe E and Pannier F. Sclerotherapy in venous malformation. Phlebology 2013; 28: 188-191.

13. Lee BB, Laredo J, Kim YW, et al. Congenital vascular malformations: general treatment principles. Phlebology 2007; 22: 258-263.

14. Greene AK and Alomari AI. Management of venous malformations. Clin Plast Surg 2011; 38: 83-93.

15. Zhang J, Li HB, Zhou SY, et al. Comparison between absolute ethanol and bleomycin for the treatment of venous malformation in children. Exp Ther Med 2013; 6: 305-309.

16. Mulliken J, Burrows $\mathrm{P}$ and Fishman S. Endovascular methods for the treatment of vascular anomalies. In: Gemmete et al. (eds) Vascular anomalies, hemangiomas and malformations, 2nd ed. USA: Oxford University Press, 2013.

17. Cavezzi A and Parsi K. Complications of foam sclerotherapy. Phlebology 2012; 27: 46-51.

18. Barranco-Pons R, Burrows PE, Landrigan-Ossar M, et al. Gross hemoglobinuria and oliguria are common transient complications of sclerotherapy for venous malformations: review of 475 procedures. AJR Am J Roentgenol 2012; 199: 691-694.

19. Hanafi M, Orliaguet G, Meyer P, et al. Pulmonary embolism in sclerotherapy for a venous malformation in a child under general anesthesia. Ann Fr Anesth Reanim 2001; 20: 556-558.

20. Marrocco-Trischitta MM, Guerrini P, Abeni D, et al. Reversible cardiac arrest after polidocanol sclerotherapy of peripheral venous malformation. Dermatol Surg 2002; 28: 153-155.

21. Chapot R, Laurent A, Enjolras O, et al. Fatal cardiovascular collapse during ethanol sclerotherapy of a venous malformation. Interv Neuroradiol 2002; 8: 321-324.

22. Zochowski CG, Salgado CJ and Jamali AA. Extensive muscle necrosis and infection following treatment of a lower extremity vascular malformation with Sotradecol and absolute ethanol. Blood Coagul Fibrinolysis 2010; 21: 480-486.

23. Lee BB. Advanced management of congenital vascular malformations (CVM). Int Angiol 2002; 21: 209-213.

24. Tan KT, Kirby J, Rajan DK, et al. Percutaneous sodium tetradecyl sulfate sclerotherapy for peripheral venous vascular malformations: a single-center experience. $J$ Vasc Intervention Radiol 2007; 18: 343-351.

25. Orlando JL, Caldas JG, Campos HG, et al. Outpatient percutaneous treatment of deep venous malformations using pure ethanol at low doses under local anesthesia. Clinics (Sao Paulo) 2010; 65: 837-840.

26. Orlando JL, Caldas JG, Campos HG, et al. Ethanol sclerotherapy of superficial venous malformation: a new procedure. Dermatology 2010; 220: 376-380.

27. Gulsen F, Cantasdemir M, Solak S, et al. Percutaneous sclerotherapy of peripheral venous malformations in pediatric patients. Pediatr Surg Int 2011; 27: 1283-1287.

28. Schumacher M, Dupuy P, Bartoli J, et al. Treatment of venous malformations: First experience with a new sclerosing agent - A multicenter study. Eur J Radiol 2011; 80: e366-e372.

29. Hoque S and Das BK. Treatment of venous malformations with ethanolamine oleate: a descriptive study of 83 cases. Pediatr Surg Int 2011; 27: 527-531.

30. Bowman J, Johnson J, McKusick M, et al. Outcomes of sclerotherapy and embolization for arteriovenous and venous malformations. Semin Vasc Surg 2013; 26: 48-54.

31. Clavien PA, Barkun J, de Oliveira ML, et al. The Clavien-Dindo classification of surgical complications: five-year experience. Ann Surg 2009; 250: 187-196. 
32. Dindo D, Demartines $\mathrm{N}$ and Clavien PA. Classification of surgical complications: a new proposal with evaluation in a cohort of 6336 patients and results of a survey. Ann Surg 2004; 240: 205-213.

33. Castren E, Aronniemi J, Klockars T, et al. Complications of sclerotherapy for 75 head and neck venous malformations. Eur Arch Otorhinolaryngol 2016; 273: 1027-1036.

34. Kollipara R, Dinneen L, Rentas KE, et al. Current classification and terminology of pediatric vascular anomalies. AJR Am J Roentgenol 2013; 201: 1124-1135.

35. Alomari A and Dubois J. Interventional management of vascular malformations. Tech Vasc Interv Radiol 2011; 14: 22-31.

36. Barranco-Pons R, Burrows PE, Landrigan-Ossar M, et al. Gross hemoglobinuria and oliguria are common transient complications of sclerotherapy for venous malformations: review of 475 procedures. AJR Am J Roentgenol 2012; 199: 691-694.

37. Dompmartin A, Ballieux F, Thibon P, et al. Elevated Ddimer level in the differential diagnosis of venous malformations. Arch Dermatol 2009; 145: 1239-1244.

38. Alomari AI, Spencer SA, Arnold RW, et al. Fibro-adipose vascular anomaly: clinical-radiologic-pathologic features of a newly delineated disorder of the extremity. J Pediatr Orthop 2014; 34: 109-117.

39. Fujiki M, Kurita M, Ozaki M, et al. Detrimental influences of intraluminally-administered sclerotic agents on surrounding tissues and peripheral nerves: an experimental study. J Plast Surg Hand Surg 2012; 46: 145-151.

40. Qiu Y, Chen H, Lin X, et al. Outcomes and complications of sclerotherapy for venous malformations. Vasc Endovascular Surg 2013; 47: 454-461. 